\title{
On a problem of R. C. Baker
}

\author{
by \\ R. NAIR (Liverpool)
}

1. Introduction. For a real number $y$, let $\langle y\rangle$ denote its fractional part. We will use $L$ to denote the collection of Lebesgue integrable functions on $[0,1)$ and $M$ to denote the bounded measurable functions on $[0,1)$. Let $\mathcal{A}$ be a collection of Lebesgue measurable functions on the interval $[0,1)$. Following [B], $[\mathrm{M}]$ we say that a strictly increasing sequence $\left(a_{k}\right)_{k=1}^{\infty}$ of natural numbers is an $\widehat{\mathcal{A}}$ sequence if for each $f$ in $\mathcal{A}$ we have

$$
\lim _{k \rightarrow \infty} \frac{1}{a_{k}} \sum_{j=1}^{a_{k}} f\left(\left\langle x+j / a_{k}\right\rangle\right)=\int_{0}^{1} f(t) d t
$$

almost everywhere with respect to Lebesgue measure. We say that $\left(a_{k}\right)_{k=1}^{\infty}$ is an $\mathcal{A}^{*}$ sequence if for each $f$ in $\mathcal{A}$,

$$
\lim _{N \rightarrow \infty} \frac{1}{N} \sum_{k=1}^{N} f\left(\left\langle a_{k} x\right\rangle\right)=\int_{0}^{1} f(t) d t
$$

almost everywhere with respect to Lebesgue measure. Examples of sequences that are both $\widehat{M}$ and $M^{*}$ appear in [B], [M], though the study of each class has an independent history going back to $[\mathrm{J}]$ and $[\mathrm{K}]$ respectively. See also $[\mathrm{E}],[\mathrm{S}]$. In this paper, in answer to a question raised in $[\mathrm{B}]$, we show that $a_{k}=2^{2^{k}}$ is an $\widehat{L}$ sequence but not an $M^{*}$ sequence. Evidently $\widehat{L} \subseteq \widehat{M}$.

As is often the case in this subject a statement's verification is straightforward given that we have isolated the right general principle, and difficult without it. To show that $a_{n}=2^{2^{k}}$ is an $\widehat{L}$ sequence, we recall B. Jessen's theorem $[\mathrm{J}]$.

Theorem A. Suppose that $\left(a_{k}\right)_{k=1}^{\infty}$ is a strictly increasing sequence of natural numbers such that $a_{k}$ divides $a_{k+1}$ for each $k$. Suppose also that $f$

2000 Mathematics Subject Classification: 11K06, 11K41, 26A42, 28D05.

Key words and phrases: Riemann sums, Lebesgue integrals, strong uniform distribution. 
is a Lebesgue integrable function on $[0,1)$. Then

$$
\lim _{k \rightarrow \infty} \frac{1}{a_{k}} \sum_{j=1}^{a_{k}} f\left(\left\langle x+j / a_{k}\right\rangle\right)=\int_{0}^{1} f(t) d t
$$

almost everywhere with respect to Lebesgue measure.

It is worthwhile to note that Jessen's theorem is a consequence of J. L. Doob's decreasing martingale theorem [D]. We now show that $a_{k}=2^{2^{k}}$ is not an $M^{*}$ sequence. Let $\left(\mu_{N}\right)_{N=1}^{\infty}$ denote a sequence of probability measures on the integers. We call the sequence $\left(\mu_{N}\right)_{N=1}^{\infty}$ dissipative if

$$
\lim _{N \rightarrow \infty} \mu_{N}(k)=0 \quad \text { for all integers } k .
$$

Suppose that we have a set $X$, a $\sigma$-algebra $\mathcal{B}$ of its subsets, and a measure $\mu$ on $X$ which is measurable with respect to $\mathcal{B}$. Suppose that $T$ is map from $X$ to itself. For $A$ in $\mathcal{B}$ set $T^{-1} A=\{x: T x \in A\}$. We call the map $T$ measurable if $T^{-1} A$ is in $\mathcal{B}$ when $A$ is; and we call it measure preserving if $\mu\left(T^{-1} A\right)=\mu(A)$ for all $A$ in $\mathcal{B}$. We call the quadruple $(X, \mathcal{B}, \mu, T)$ a dynamical system if it is measurable and measure preserving. A dynamical system $(X, \mathcal{B}, \mu, T)$ is called ergodic if $\mu\left(A \triangle T^{-1} A\right)=0$ implies that $\mu(A)$ is either zero or one. Here for two sets $A$ and $B$ we have used $A \triangle B$ to denote their symmetric difference.

For a sequence $\left(\mu_{N}\right)_{N=1}^{\infty}$ of probability measures on the integers and $f$ in $L^{1}(X, \mathcal{B}, \mu)$,

$$
\left(\mu_{N} f\right)(x)=\sum_{k=-\infty}^{\infty} \mu_{N}(k) f\left(T^{k} x\right) \quad(N=1,2, \ldots) .
$$

Given $\delta>0$, a sequence of probability measures $\left(\mu_{N}\right)_{N=1}^{\infty}$ is called $\delta$ sweeping out if for all ergodic dynamical systems $(X, \mathcal{B}, \mu, T)$ and all $\varepsilon>0$ there exists $E$ in $\mathcal{B}$ such that $\mu(E) \leq \varepsilon$ and

$$
\limsup _{N \rightarrow \infty} \mu_{N} I_{E}(x) \geq \delta
$$

$\mu$-almost everywhere. Here $I_{E}$ denotes the indicator function of the set $E$.

We need the following theorem proved in [Ro].

Theorem B. Suppose that $S=\left(b_{k}\right)_{k=1}^{\infty}$ is a sequence of integers with

$$
\inf _{k \geq 1} \frac{b_{k+1}}{b_{k}}>1
$$

and that each measure $\mu_{N}(N=1,2, \ldots)$ has support contained in $S$. Then $\left(\mu_{N}\right)_{N=1}^{\infty}$ is $\delta$-sweeping out for some $\delta>0$.

We use this theorem by applying it to the setting where

$$
b_{k}=2^{k} \quad(k=1,2, \ldots),
$$




$$
\mu_{N}=\frac{1}{N} \sum_{k=1}^{N} \delta_{b_{k}} \quad(N=1,2, \ldots)
$$

for delta measures $\delta_{a}$ defined by

$$
\delta_{a}(A)= \begin{cases}1 & \text { if } a \in A, \\ 0 & \text { if } a \notin A,\end{cases}
$$

defined on the integers, $X=[0,1), \mathcal{B}$ is the Lebesgue $\sigma$-algebra, $\mu$ is Lebesgue measure on $[0,1)$ and the map $T$ is defined by $T x=\langle 2 x\rangle$. The fact that $T$ both preserves Lebesgue measure on $[0,1)$ and is ergodic is proved in [W]. For a Lebesgue measurable set $A$ let $|A|$ denote its Lebesgue measure. The upshot of this is that there exists $\delta>0$ such that for any $\varepsilon>0$, there exists a Lebesgue measurable set $E$ contained in $[0,1)$ such that $|E|<\varepsilon$ and

$$
\limsup _{N \rightarrow \infty} \frac{1}{N} \sum_{k=1}^{N} I_{E}\left(\left\langle 2^{2^{k}} x\right\rangle\right) \geq \delta>0
$$

almost everywhere with respect to Lebesgue measure.

Thus of course $a_{k}=2^{2^{k}}(k=1,2, \ldots)$ is not an $M^{*}$ sequence. It would be interesting to know if we could choose $\delta=1$. Evidently in the previous argument Lebesgue measurable can be replaced by Borel measurable everywhere.

Plainly for any strictly increasing sequence $\left(c_{k}\right)_{k=1}^{\infty}$ of natural numbers, the sequence $a_{k}=2^{c_{k}}(k=1,2, \ldots)$ is an $\widehat{L}$ sequence. Given $p$ in $[1, \infty)$ it is possible to give strictly increasing sequences $\left(c_{k}\right)_{k=1}^{\infty}$ of integers such that $a_{k}=2^{c_{k}}(k=1,2, \ldots)$ is in $\left(L^{p}\right)^{*}$ but not in $\left(L^{q}\right)^{*}$ for any $q<p$. Here $L^{p}$ denotes the space of Lebesgue measurable functions on $[0,1)$ whose $p$ th powers are Lebesgue integrable. This observation relies on a result of K. Reinhold-Larsson [RL].

Theorem C. Given $p$ in $[1, \infty)$, there exists a strictly increasing sequence $\left(c_{k}\right)_{k=1}^{\infty}$ of natural numbers such that for every dynamical system $(X, \mathcal{B}, \mu, T)$ and every function $f$ in $L^{p}(X, \mathcal{B}, \mu)$ there exists $C_{p}>0$ such that if

$$
M f(x)=\left|\sup _{N \geq 1} \sum_{k=1}^{N} f\left(T^{c_{k}} x\right)\right|,
$$

then

$$
\mu(\{x \in X: M f(x)>\alpha\}) \leq \frac{C_{p}}{\alpha^{p}}\|f\|_{p}
$$

where

$$
\|f\|_{p}=\left(\int_{X}|f|^{p}(x) d \mu\right)^{1 / p} .
$$


Also if $1 \leq q<p$ then there exists $f$ in $L^{q}(X, \mathcal{B}, \mu)$ such that

$$
\lim _{N \rightarrow \infty} \frac{1}{N} \sum_{k=1}^{N} f\left(T^{c_{k}} x\right)
$$

does not have a finite limit for almost all $x$ with respect to $\mu$.

Choosing $X=[0,1), \mathcal{B}$ to be the Lebesgue $\sigma$-algebra, $\mu$ the Lebesgue measure and $T x=\langle 2 x\rangle$ and using Theorem $\mathrm{C}$ as before shows that $a_{k}=2^{c_{k}}$ $(k=1,2, \ldots)$ does not belong to $\left(L^{q}\right)^{*}$. To show that $\left(2^{c_{k}}\right)_{k=1}^{\infty}$ is in $\left(L^{p}\right)^{*}$ we need to show that

$$
\lim _{N \rightarrow \infty} \frac{1}{N} \sum_{k=1}^{N} f\left(\left\langle 2^{c_{k}} x\right\rangle\right)=\int_{0}^{1} f(t) d t
$$

almost everywhere with respect to Lebesgue measure. By a classical theorem of $\mathrm{H}$. Weyl $[\mathrm{Wy}]$ this is known for continuous functions on $[0,1)$. Suppose that $\left(f_{n}\right)_{n=1}^{\infty}$ is a sequence of continuous functions on $[0,1)$ converging to $f$ in $L^{p}$ norm. This means that there exists a subsequence $\left(n_{k}\right)_{k=1}^{\infty}$ such that

$$
\sum_{k=1}^{\infty} \int_{0}^{1}\left|f-f_{n_{k}}\right|^{p}(x) d x<\infty
$$

which implies that

$$
\sum_{k=1}^{\infty}\left|f-f_{n_{k}}\right|^{p}(x)<\infty
$$

almost everywhere with respect to Lebesgue measure on $[0,1)$. Thus for every $\varepsilon>0$, there exists a sequence of functions $\left(f_{\varepsilon, k}\right)_{k=1}^{\infty}$ such that

$$
\left\|f-f_{\varepsilon, k}\right\|_{p}^{p} \leq \varepsilon^{2 k}
$$

and $f_{\varepsilon, k}$ tends to $f$ as $k$ tends to infinity almost everywhere with respect to Lebesgue measure on $[0,1)$. Notice that

$$
M(f+g) \leq M(f)+M(g) .
$$

Let

$$
E_{\varepsilon, k}:=\left\{x \in[0,1): M\left(f-f_{\varepsilon, k}\right)(x)>\varepsilon^{k / p}\right\}
$$

and note from Theorem $\mathrm{C}$ that

$$
\mu\left(E_{\varepsilon, k}\right) \leq C_{p}\left(\frac{1}{\varepsilon}\right)^{k} \int_{E_{\varepsilon, k}}\left|f-f_{\varepsilon, k}\right|^{p}(x) d x \leq C_{p}\left(\frac{1}{\varepsilon}\right)^{k} \varepsilon^{2 k}=C_{p} \varepsilon^{k} .
$$

Let $a_{N}(f, x)$ denote $\frac{1}{N} \sum_{l=1}^{N} f\left(\left\langle 2^{c_{l}} x\right\rangle\right)$. Now

$$
a_{N}(f, x)=a_{N}\left(f-f_{\varepsilon, k} x\right)+a_{N}\left(f_{\varepsilon, k}, x\right) .
$$


This means that

$$
\left|a_{N}(f, x)-\int_{0}^{1} f(t) d t\right| \leq\left|a_{N}\left(f-f_{\varepsilon, k}, x\right)\right|+\left|a_{N}\left(f_{\varepsilon, k}, x\right)-\int_{0}^{1} f(t) d t\right|
$$

almost everywhere with respect to Lebesgue measure on $[0,1)$. Thus

$$
\begin{aligned}
& \limsup _{N \rightarrow \infty}\left|a_{N}(f, x)-\int_{0}^{1} f(t) d t\right| \\
& \quad \leq \limsup _{N \rightarrow \infty}\left|a_{N}\left(f-f_{\varepsilon, k}, x\right)\right|+\left|\int_{0}^{1}\left(f-f_{\varepsilon, k}\right)(t) d t\right|,
\end{aligned}
$$

which is

$$
\leq M\left(f-f_{\varepsilon, k}\right)(x)+\int_{0}^{1}\left|f-f_{\varepsilon, k}\right|(t) d t .
$$

Therefore as $N$ tends to infinity we know that $a_{N}(f, x)$ tends to $\int_{0}^{1} f(t) d t$ for all $x$ in $E_{\varepsilon}=\bigcup_{n=1}^{\infty} E_{\varepsilon, n}$. Let $B_{\varepsilon}$ be the null set off which $f_{\varepsilon, k}$ tends to $f$ as $k \rightarrow \infty$. This means that

$$
\lambda\left(E_{\varepsilon} \cup B_{\varepsilon}\right) \leq \sum_{n=1}^{\infty} \lambda\left(E_{\varepsilon, k}\right) \leq C_{p} \sum_{k=1}^{\infty} \varepsilon^{k}=\frac{C_{p} \varepsilon}{1-\varepsilon} .
$$

Letting $\varepsilon$ tend to zero shows that $\left(2^{c_{k}}\right)_{k=1}^{\infty}$ is in $\left(L^{p}\right)^{*}$ for finite $p$.

\section{References}

[B] R. C. Baker, Riemann sums and Lebesgue integrals, Quart. J. Math. Oxford Ser. (2) 27 (1976), 191-198.

[D] J. L. Doob, Stochastic Processes, Wiley, 1953.

[E] P. Erdős, On the strong law of large numbers, Trans. Amer. Math. Soc. 67 (1949), 51-56.

[J] B. Jessen, On the approximation of Lebesgue integrals by Riemann sums, Ann. of Math. (2) 35 (1934), 248-251.

[K] A. Khinchin, Ein Satz über Kettenbrüche, mit arithmetischen Anwendungen, Math. Z. 18 (1923), 289-306.

[M] J. M. Marstrand, On Khinchin's conjecture about strong uniform distribution, Proc. London Math. Soc. 21 (1970), 540-556.

[RL] K. Reinhold-Larsson, Discrepancy of behaviour of perturbed sequences in $L^{p}$ spaces, Proc. Amer. Math. Soc. 120 (1994), 865-874.

[Ro] J. Rosenblatt, Universally bad sequences in ergodic theory, in: Almost Everywhere Convergence II, Academic Press, 1991, 227-245.

[S] R. Salem, Sur les sommes Riemanniennes des fonctions sommables, Mat. Tidsskr. B. $1948,60-62$.

[W] P. Walters, Introduction to Ergodic Theory, Grad. Texts in Math. 79, Springer, 1981. 
[Wy] H. Weyl, Über die Gleichverteilung von Zahlen mod. Eins, Math. Ann. 77 (1916), 313-352.

Mathematical Sciences

University of Liverpool

Liverpool L69 7ZL, U.K.

E-mail: nair@liverpool.ac.uk

Received on 12.4.2002

and in revised form on 17.9.2002 